\title{
Preface
}

\section{Intelligent Systems and Knowledge Management (Part II)}

\author{
Dimitris Kanellopoulos, Sotiris Kotsiantis and Panagiotis Pintelas*
}

This is the second special issue which focuses on "Intelligent Systems and Knowledge Management". Intelligent systems refer broadly to computer embedded or controlled systems, machines and devices that possess a certain degree of intelligence. Much work in the area can be fairly characterized as integration on different levels of several technology directions - intelligent control, artificial intelligence, decision support, soft computing, knowledge discovery, data mining, data fusion, knowledge engineering, ontologies, machine learning and neural networks - with challenging applications to complex systems. Knowledge management $(\mathrm{KM})$ technology is used to rapidly capture, organize and deliver large amounts of corporate knowledge for business and/or e-learning processes.

This special issue of the Journal of Computational Methods in Sciences and Engineering (JCMSE) invited authors to submit their original work that communicates current research on intelligent systems, regarding both the theoretical and methodological aspects, as well as various applications of intelligent approaches to many real world problems from science, technology or business.

The first paper of this special issue proposes a collaborative ranking mechanism for efficient and personalized Internet search service provisioning. The second paper studies the perception of musical structure combined with mirror neuron (MN) system activation. In the light of this structure, let us be more descriptive.

Traditional knowledge management is facing new problems triggered by the Web such as information overload, inefficient keyword searching, heterogeneous information integration and geographicallydistributed intranet problems etc.). In the first paper, M. Louta, I. Anagnostopoulos, I. Maglogiannis, and A. Michalas propose enhancements to the sophistication of the functionality that can be offered by search engine services. The authors propose a metasearch third-party result ranking mechanism, which is capable of adapting over the continuous changes that occur on the web, providing in parallel personalized information acquisition considering the user's navigation behaviour. Transparency is achieved for both personalization and web evolution adaptation mechanisms, requiring virtually none effort from the user's part. In essence, the proposed meta search engine rates, re-organises and combines the results acquired from search services for a specific user information resource request in accordance with a weighted combination of a performance related factor (tightly related to the ranking of the web result as given by the search engine service) and a reliability related factor (corresponding to the user satisfaction stemming from the specific web result that he/she browses), while the performance of each search engine with

\footnotetext{
${ }^{*}$ Corresponding author. E-mail: pintelas@ math.upatras.gr.
} 
respect to adequately adapting to the web evolution is taken into account. For the evaluation of the web results reliability, a collaborative reputation mechanism is utilized, which helps estimating their quality and predicting their future usability, taking into account their past performance in consistently satisfying user expectations. A set of results indicative of the efficiency of their proposed scheme is provided. The Internet search services used were Google, MSN and Yahoo!

The second paper, by Panagiotis Doulgeris, Stelios Hadjidimitriou, Konstantinos Panoulas, Leontios Hadjileontiadis and Stavros Panas, studies the perception of musical structure combined with mirror neuron (MN) system activation. The main idea is to analyze EEGs recordings, and especially the Murhythm that relates to the activation of MN. The authors consider the response of mirror neuron (MN) cells to intention and 'motion' involved in musical structures. They designed three experiments (viz., 2 experiments targeted to intention and 1 to motion) which resulted to interesting outcomes concerning the role of MNs to the way we see knowledge scaffolding in music perception.

The list of the papers follows:

- M. Louta, I. Anagnostopoulos, I. Maglogiannis, A. Michalas. Towards a collaborative ranking mechanism for efficient and personalized Internet search service provisioning.

- P. Doulgeris, S. Hadjidimitriou, K. Panoulas, L. Hadjileontiadis, and S. Panas. Bispectral EEG analysis for knowledge scaffolding in music perception: A mirror neurons-based approach.

The guest editors wish to thank Professor T. Simos (Editor-in-Chief of the Journal of Computational Methods in Sciences and Engineering) for providing the opportunity to edit this special issue on Intelligent Systems and Knowledge Management. We would also like to thank the referees who have critically evaluated the papers within the short stipulated time. Finally, we hope that the papers in this issue will stimulate further progress in this direction. 